\title{
No efficacy of transcranial direct current stimulation on chronic migraine with medication overuse: A double blind, randomised clinical trial
}

Cephalalgia

0 (0) $1-10$

(C) International Headache Society 2020

Article reuse guidelines:

sagepub.com/journals-permissions DOI: $10.1177 / 0333102420931050$ journals.sagepub.com/home/cep @SAGE

\author{
Licia Grazzi', Susanna Usai', Nadia Bolognini ${ }^{2,3}$, \\ Eleonora Grignani', Emanuela Sansone', Irene Tramacere ${ }^{4}$, \\ Angelo Maravita $^{2}$ and Giuseppe Lauria ${ }^{1,5}$ (1)
}

\begin{abstract}
Background: Transcranial direct current stimulation was suggested to provide beneficial effects in chronic migraine, a condition often associated with medication overuse for which no long-term therapy is available.

Methods: We conducted a randomised controlled trial to assess long-term efficacy of transcranial direct current stimulation. Adults diagnosed with chronic migraine and medication overuse were assigned to receive in a I:I:I ratio anodal, cathodal, or sham transcranial direct current stimulation daily for five consecutive days, along with standardised drug withdrawal protocol. Primary outcome was 50\% reduction of days of headache per month at 12 months. Co-secondary outcomes were $50 \%$ reduction of days of headache per month at 6 months, reduction of analgesic intake per month, and change in disability and quality of life, catastrophising, depression, state and trait anxiety, dependence attitude and allodynia intensity. Patients were not allowed to take any migraine prophylaxis drug for the entire study period.

Results: We randomly allocated 135 patients to anodal (44), cathodal (45), and sham (46) transcranial direct current stimulation. At 6 and 12 months, the percentage of reduction of days of headache and number of analgesics per month ranged between $48.5 \%$ and $64.7 \%$, without differences between transcranial direct current stimulation (cathodal, anodal, or the results obtained from the two arms of treatment, anodal plus cathodal) and sham. Catastrophising attitude significantly reduced at 12 months in all groups. There was no difference for the other secondary outcomes. Conclusions: Transcranial direct current stimulation did not influence the short and long-term course of chronic migraine with medication overuse after acute drug withdrawal. Behavioral and educational measures and support for patients' pain management could provide long-term improvement and low relapse rate.
\end{abstract}

Trial registration number NCT04228809

Keywords

Non-invasive stimulation, headache, pain management, drug withdrawal

Date received: 28 March 2020; revised: 27 April 2020; 7 May 2020; accepted: 10 May 2020

\footnotetext{
'Neuroalgology Unit, Headache Centre, Fondazione IRCCS Istituto Neurologico "Carlo Besta", Milan, Italy

${ }^{2}$ Department of Psychology and Milan Center for Neuroscience, University of Milano-Bicocca, Milan, Italy

${ }^{3}$ IRCCS Istituto Auxologico Italiano, Neuropsychology Laboratory, Milan, Italy
}

${ }^{4}$ Department of Research and Clinical Development, Scientific Directorate, Fondazione IRCCS Istituto Neurologico "Carlo Besta", Milan, Italy

${ }^{5}$ Department of Biomedical and Clinical Sciences "Luigi Sacco", University of Milan, Milan, Italy

\section{Corresponding author:}

Licia Grazzi, Neuroalgology Unit, Headache Center IRCCS Fondazione Istituto Neurologico "Carlo Besta" via Celoria, II, 20133 Milan, Italy. Email: licia.grazzi@istituto-besta.it 


\section{Introduction}

Migraine is a complex clinical syndrome encompassing a variety of somatic and autonomic symptoms, among which pain is the most relevant and drives diagnosis and treatments. Chronic migraine (CM) is a subtype that the International Headache Society (IHS) classified as headache with a frequency at least for 15 days per month with eight migraine days per month (1). It affects about $2 \%$ of the migraine population and is a particularly disabling condition with huge physical and emotional burden and high direct and indirect costs (2). CM patients are difficult to treat also because of the high frequency of psychiatric comorbidities, like anxiety and depression, and available pharmacological therapies often cannot provide satisfactory pain relief. The regular use of medications for the treatment of primary migraine episodes can lead to an increased frequency and intensity of migraine attacks (2). Such association with medication overuse (MO) makes the overall treatment even more challenging. Drug withdrawal is considered the first step for adequately managing MO patients (2). However, only a few studies reported findings at 12 months and none in the absence of pharmacological prophylaxis $(3,4)$.

In recent years, the efficacy of neurostimulation techniques to treat migraine has been tested using different approaches in several clinical studies, with inconclusive results due to different reasons: No standardised protocols of application, heterogeneous groups of patients, short follow-up, poor criteria for clinical efficacy measures $(5,6)$. Transcranial direct current stimulation (tDCS) is a technique of brain modulation that in the last few years has been used in various neurological and psychiatric disorders (7). It has the advantage of allowing a reliable placebo condition to test its efficacy in randomised trials (7), and it has been proposed to provide beneficial effects in different types of chronic pain (8-10). No conclusions could be drawn, mainly because of the poor quality of most of the clinical studies $(11,12)$.

Transcranial direct current stimulation causes polarity-dependent shifts of the resting membrane potential and consequently could change neuronal excitability at the site of stimulation and in the connected areas. If the stimulation lasts long enough, tDCS might induce neurophysiological and behavioural aftereffects, which are thought to depend on synaptic plasticity (7).

Given the results of previous studies suggesting that a technique able to modulate pain-related neural networks, such as tDCS, could successfully treat CM $(5,6,13)$ and the emphasised need for confirmatory trials with more robust methodology (14-17), we designed a placebo-controlled, double-blind, randomised clinical trial (RCT) combined with an ensuing open-label study to assess, over 1-year follow-up, the short-, mid- and long-term efficacy of tDCS in patients diagnosed with CM and MO after acute drug withdrawal and no migraine prophylaxis.

\section{Methods}

\section{Trial protocol}

This was a monocentre study carried out at the Headache Centre of the IRCCS Foundation "Carlo Besta" Neurological Institute of Milan, Italy in collaboration with the Department of Psychology of the Milano Bicocca University, Milan, Italy. The trial was performed in accordance with the declaration of Helsinki and approved by the local Ethic Committee on July 2015.

Participants were assessed for eligibility during a screening visit in which inclusion and exclusion criteria were applied (Figure 1). Upon inclusion, participants were randomly assigned in 1:1:1 ratio to anodal tDCS, cathodal tDCS, or sham stimulation, which started the same day as the standardised acute drug withdrawal.

At the end of the recruitment for the RCT, we started the open-label trial in which participants were assigned to standardised acute drug withdrawal. This arm was added to provide a pragmatic, non-parallel comparison with acute drug withdrawal, which is considered the most effective treatment of $\mathrm{CM}$ with $\mathrm{MO}$ (18). The reason why it was not added as one further arm of the double-blind part of the study is because results would have been biased by the lack of any active or sham tDCS procedure, and therefore would have not been comparable. Even though this approach had the intrinsic limitation of a non-parallel analysis, we thought it could have provided a clinically meaningful comparison between the results from the RCT and the current clinical practice, while ensuring the integrity of the RCT.

\section{Trial participants}

Participants both in the RCT and the ensuing open label study were adults diagnosed with $\mathrm{CM}$ and $\mathrm{MO}$ according to the IHS criteria (1), who must have failed at least two pharmacological prophylaxis therapies. Diagnosis was confirmed on the basis of a daily headache diary that all participants filled out in the last month prior to the enrolment. All participants gave written informed consent. Patients were not involved in the design, conduct, reporting, or dissemination plans for our research.

Exclusion criteria were a known diagnosis of major depression or other major psychiatric disorders 


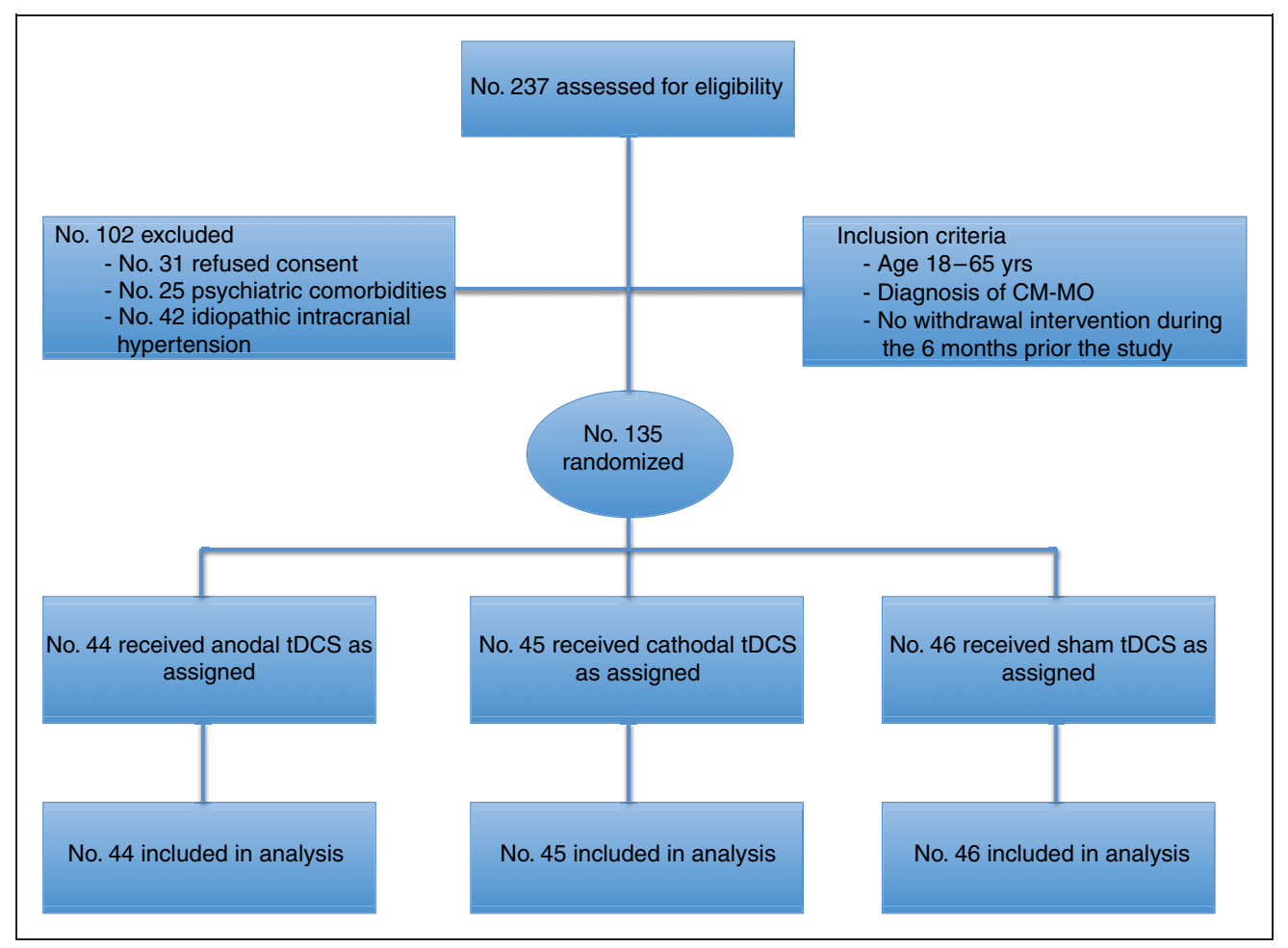

Figure I. Trial profile.

identified after psychiatric consultation, having a cardiac pacemaker, clips for previous head surgery, cochlear implant, a history of epilepsy, known idiopathic intracranial hypertension, harmful alcohol consumption, and pregnancy.

\section{Sample size calculation}

Based on an expected effect of $50 \%$ in the sham group at the 12 -month visit (i.e. $50 \%$ of patients with a reduction of at least $50 \%$ of days of migraine/month) assuming alpha $=0.1$ and power $=80 \%$ to determine a significant absolute difference of at least $30 \%$ (i.e. an expected $80 \%$ of patients with a reduction of at least $50 \%$ of days of migraine/month) between any of the groups (i.e. cathodic versus sham or anodic versus sham), and assuming a loss to follow-up of $15 \%$, we estimated that 135 patients (45 per arm) should be recruited and followed in the RCT for 12 months.

\section{Randomisation and blindness}

Participants in the RCT were assigned to the treatment arms according to a centralised randomisation schedule. The randomisation schedule was prepared in the data management system by an independent trial statistician. The two study neurologists (LG and SU), who enrolled and followed the patients, were not involved in the randomisation procedure and were blind to the treatment condition. The two technicians (ES and EG), who performed the tDCS procedures, were aware of the treatment conditions but were not involved in any of the clinical assessments.

\section{Treatments}

The day of randomisation, participants started a standardised acute drug withdrawal protocol (4) with intravenous administration of dexamethasone $4 \mathrm{mg}$ and ademetionine $200 \mathrm{mg}$ in saline solution, and oral bromazepam $1.5 \mathrm{mg}$ three times daily, for five consecutive days. The same day, they started the tDCS procedure based on allocation (anodal, cathodal, or sham). Both acute drug withdrawal and tDCS were performed daily for five consecutive days.

The tDCS was delivered with a battery-driven constant current stimulator (BrainStim, EMS srl, Bologna, Italy; http://brainstim.it) through a pair of surface saline-soaked sponge electrodes $(35 \mathrm{~cm}, 7 \times 5 \mathrm{~cm})$ placed on the patient's scalp. The stimulation was applied daily, for a duration of 20 minutes, current intensity of $2 \mathrm{~mA}$, fade in/fade out phases $=10 \mathrm{sec}$, for five consecutive days during the withdrawal program. Patients were randomised to receive anodal, cathodal or sham tDCS; in every case, the active electrode was placed over the primary motor cortex of the right 
hemisphere (site C4 of the 10/20 EEG system) with the reference electrode placed over the contralateral supraorbital area.

Motor cortex stimulation is thought to exert analgesic effects by activating descending pathways, hence restoring inhibitory control of nociceptive transmission. The analgesic effects of tDCS occur through various neural circuits involved in sensori-discriminative, cognitive, and emotional aspect of chronic pain (19). Although theoretically having opposite effects on cortical excitability, with anodal tDCS increasing cortical excitability and cathodal tDCS decreasing it, both polarities were found to be effective on pain (7). With regard to chronic pain syndromes, also including migraine, sham-controlled studies showed some positive results for both anodal and cathodal tDCS $(5,17,20)$. Hence, here we have further explored, and directly compared, the effects of both types of stimulations (anodal and cathodal tDCS) of the motor cortex. As far as the parameters of tDCS stimulation and treatment duration, we used a typical protocol for pain treatment (21), consisting of five daily tDCS applications for 20 minutes, at $2 \mathrm{~mA}$ of current intensity.

For sham tDCS, the stimulator was turned off after $30 \mathrm{sec}$. The sham and the real tDCS modes (anodal/ cathodal) were activated through a code by one of the experimenters (ES), who did not participate in data collection and patients' clinical outcome.

Participants in the open-label trial received the standardised acute drug withdrawal above described for five consecutive days, then entered the follow-up, and no application of tDCS was provided.

Participants both in the RCT and open-label trial were not allowed to start any prophylaxis treatment for migraine during the 12-month study period.

\section{Efficacy end points}

The primary outcome was the proportion of patients with a reduction of at least $50 \%$ of days of migraine per month recorded by a specific Daily Headache Diary at 12-month visit.

The secondary outcomes were the reduction of days of migraine per month, the reduction of analgesic intake per month, and the change in disability and quality of life (Migraine Disability Assessment, MIDAS; Headache Impact Test, HIT-6), catastrophising (Pain Catastrophizing Scale), depression (Beck Depression Inventory) and state and trait anxiety (Spielberger questionnaires), dependence attitude (Leeds questionnaire) and allodynia intensity (Allodynia Symptoms Checklist, ASC).

\section{Follow-up visits}

Participants enrolled both in the RCT and the openlabel trial underwent follow-up visits at 3, 6, 9, and 12 months after the end of drug withdrawal and tDCS treatments. All clinical data included in primary and secondary outcomes were recorded at each follow-up visit.

\section{Statistical analysis}

Primary analysis of efficacy was performed by intention-to-treat. The efficacy of tDCS was assessed by binomial regression. Repeated measures analysis of variance (ANOVA) was used in order to assess effect of treatment and difference between treatment groups in terms of decrease in number of days of migraine per month and number of analgesics taken per month throughout the 12-month study period. Differences between groups in the secondary outcome changes from baseline to 12-month visit were analysed through the ANOVA adjusted for baseline values. Statistical analysis was carried out using STATA statistical software, version 15 (StataCorp. 2017. Stata Statistical Software: Release 15. College Station, TX: StataCorp LLC).

\section{Data availability}

The data that support the findings of this study are available from the corresponding author.

\section{Results}

Between December 2015 and June 2017, we consecutively screened 237 patients. One hundred and two patients were not included because they did not give consent (31 patients), had known psychiatric comorbidities (25 patients), idiopathic intracranial hypertension (42 patients), or other comorbidities (four patients). Eventually, 135 patients were randomly allocated to the anodal tDCS arm (44 patients), the cathodal tDCS arm (45 patients), and sham arm (46 patients) (Figure 1).

Thereafter, between August 2017 and May 2018, we consecutively screened 51 patients and eventually enrolled 22 patients for the open-label study. Twentynine patients were not included for known psychiatric comorbidities (10 patients) or because they did not give consent (19 patients).

At baseline, demographic variables and age of migraine onset, days of migraine per month, and mean analgesic drug intake per month did not differ among groups (Table 1). At 12-month follow-up, the proportion of patients with a reduction of at least $50 \%$ of days of migraine per month was not significantly 
Table I. Demographic variables of patients enrolled in the randomised trial (anodal, cathodal, sham) and the open label trial (drug withdrawal). Number of days of migraine and number of analgesics refer to the month prior to the enrolment.

\begin{tabular}{llllll}
\hline & $\begin{array}{l}\text { No. of } \\
\text { patients (F/M) }\end{array}$ & $\begin{array}{l}\text { Age, } \\
\text { mean } \pm \text { SD }\end{array}$ & $\begin{array}{l}\text { Age at onset, } \\
\text { mean } \pm \text { SD }\end{array}$ & $\begin{array}{l}\text { No. of days of } \\
\text { migraine/month, } \\
\text { mean } \pm \text { SD }\end{array}$ & $\begin{array}{l}\text { No. of } \\
\text { analgesics/month, } \\
\text { mean } \pm \text { SD }\end{array}$ \\
\hline Anodal & $45(4 I / 4)$ & $47.8 \pm 10.8$ & $19.5 \pm 10.1$ & $19.5 \pm 10.1$ & $20.9 \pm 6.2$ \\
Cathodal & $44(36 / 8)$ & $47.7 \pm 13.1$ & $21.0 \pm 11.0$ & $20.9 \pm 11.0$ & $23.1 \pm 6.9$ \\
Sham & $46(37 / 9)$ & $45.5 \pm 11.3$ & $17.9 \pm 8.9$ & $17.9 \pm 8.8$ & $21.5 \pm 6.9$ \\
Drug withdrawal & $22(21 / 1)$ & $43.5 \pm 9.2$ & $19.3 \pm 7.3$ & $21.8 \pm 7.8$ & $18.7 \pm 6.7$ \\
\hline
\end{tabular}

SD: standard deviation.

Table 2. Number of days of migraine per month recorded by the Daily Headache Diary at baseline and at 6 and I2-month visit in the patients enrolled in the randomised trial (anodal, cathodal, sham) and the open label trial (drug withdrawal).

\begin{tabular}{llll}
\hline & $\begin{array}{l}\text { No. of days of } \\
\text { migraine/month } \\
\text { Baseline, } \\
\text { mean } \pm \text { SD }\end{array}$ & $\begin{array}{l}\text { No. of days of } \\
\text { migraine/month } \\
\text { 6-month follow-up, } \\
\text { mean } \pm \text { SD }\end{array}$ & $\begin{array}{l}\text { No. of days of } \\
\text { migraine/month } \\
\text { I2-month follow-up, } \\
\text { mean } \pm \text { SD }\end{array}$ \\
\hline Anodal & $20.4 \pm 6.6$ & $13.3 \pm 10.2$ & $11.0 \pm 7.5$ \\
Cathodal & $23.5 \pm 7.0$ & $11.8 \pm 8.6$ & $11.5 \pm 8.6$ \\
Sham & $21.8 \pm 6.4$ & $12.6 \pm 8.9$ & $13.0 \pm 7.8$ \\
Drug withdrawal & $19.6 \pm 7.4$ & $10.9 \pm 8.2$ & $9.9 \pm 7.4$ \\
\hline
\end{tabular}

SD: standard deviation.

Table 3. Number of analgesics taken per month at baseline and at 6 and 12-month visit in the patients enrolled in the randomised trial (anodal, cathodal, sham) and the open label trial (drug withdrawal).

\begin{tabular}{llll}
\hline & $\begin{array}{l}\text { No. of analgesics/month } \\
\text { Baseline, } \\
\text { mean } \pm \text { SD }\end{array}$ & $\begin{array}{l}\text { No. of analgesics/month } \\
\text { 6-month follow-up, } \\
\text { mean } \pm \text { SD }\end{array}$ & $\begin{array}{l}\text { No. of analgesics/month } \\
\text { I2-month follow-up, } \\
\text { mean } \pm \text { SD }\end{array}$ \\
\hline Anodal & $20.9 \pm 6.2$ & $11.4 \pm 10.2$ & $10.6 \pm 8.4$ \\
Cathodal & $23.1 \pm 6.9$ & $10.9 \pm 9.6$ & $10.4 \pm 10.4$ \\
Sham & $21.5 \pm 6.9$ & $12.7 \pm 11.6$ & $12.6 \pm 8.9$ \\
Drug withdrawal & $17.5 \pm 6.5$ & $10.3 \pm 7.6$ & $8.2 \pm 5.1$ \\
\hline
\end{tabular}

SD: standard deviation.

different between groups: $64.1 \%$ in anodal, $60.0 \%$ in cathodal, $46.3 \%$ in sham and $55.0 \%$ in the open-label arm. At 6 and 12-month follow-up, all groups showed similar reductions of days of migraine per month between tDCS (either cathodal, anodal or the results obtained from the two arms of treatment, anodal plus cathodal) and sham arms, and open label arm (Table 2). Similarly, the number of analgesics taken per month reduced at 6 and 12-month follow-up, with no significant differences between tDCS (either cathodal, anodal, or the results obtained from the two arms of treatment, anodal plus cathodal) and sham arms, and the open-label arm (Table 3). Repeated measures analysis of variance demonstrated a significant effect of any treatment with no difference between groups, both in terms of number of days of migraine per month (Figure 2(a)) and number of analgesics taken per month (Figure 2(b)). All the other outcomes improved at 12 months compared to baseline (Table 4).

Patients in both treatment groups reported no side effects from tDCS application during the course of therapy and during the follow-up.

\section{Discussion}

Our results confirm that tDCS did not influence the frequency of chronic migraine in a population of patients with medication overuse. These findings confirm the lack of efficacy of tDCS, as previously demonstrated in a cohort of patients with chronic low back 

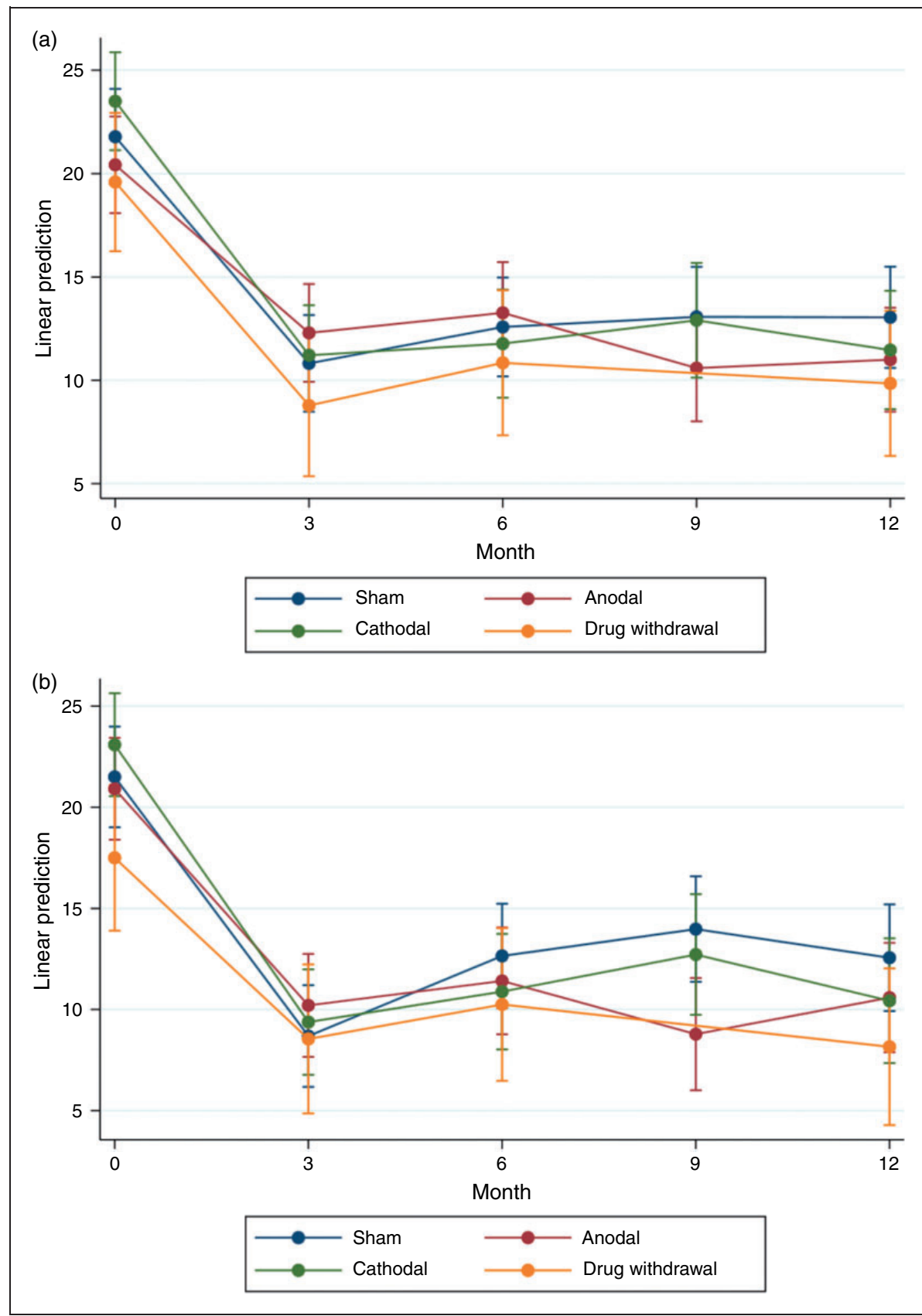

Figure 2. Repeated measures analysis of variance showing a significant effect of any treatment compared to baseline as to (a) number of days of migraine per month $(p<0.0 \mathrm{I})$ and $(b)$ number of analgesics taken per month $(p<0.0 \mathrm{I})$, with no difference between groups $(p=0.39$ and 0.12 , respectively).

pain (22). Whether tDCS might exert any positive effect in other acute pain disorders remains unaddressed.

Migraine can remain episodic or become a chronic disorder, this latter having a high burden for patients' quality of life and health system costs, mainly when complicated by MO. Unknown individual genetic backgrounds, behavioural profiles, and psychiatric comorbidities likely play key roles in driving its course. For these reasons migraine, in particular chronic migraine, remains a complex disease that is difficult to treat. In real life, most CM patients record a monthly use of 15-25 analgesics, less than 5-8 days free of migraine per month, several failed pharmacological prophylaxis, and a high rate of relapse after drug withdrawal. The design of clinical studies aiming to unravel the effect of treatments in CM should acknowledge the overall context, consider clinically meaningful changes, 
Table 4. Outcome changes from baseline to 12 -month visit recorded in the randomised controlled trial. $p$-values are from the analysis of variance (ANOVA) between groups adjusted for baseline value. Migraine Disability Assessment (MIDAS); Headache Impact Test (HIT-6); state and trait anxiety (Spielberger questionnaires (STAIS and STAIT)); depression (Beck Depression Inventory (BDI)); dependence attitude (Leeds questionnaire (LDQ)); catastrophising attitude (Pain Catastrophizing Scale (PCS)); allodynia intensity (Allodynia Symptoms Checklist (ASC)).

\begin{tabular}{|c|c|c|c|c|}
\hline Outcome & $\begin{array}{l}\text { Anodal tDCS, } \\
\text { mean } \pm S D\end{array}$ & $\begin{array}{l}\text { Cathodal tDCS, } \\
\text { mean } \pm S D\end{array}$ & $\begin{array}{l}\text { Sham, } \\
\text { mean } \pm \text { SD }\end{array}$ & $p$ \\
\hline MIDAS & $-45.4 \pm 48.4$ & $-27.8 \pm 67.5$ & $-|7.1 \pm 46|$. & 0.60 \\
\hline HIT-6 & $-4.4 \pm 9.1$ & $-4.7 \pm 8.8$ & $-3.6 \pm 6.9$ & 0.28 \\
\hline STAIS & $-2.3 \pm 13.1$ & $-1.5 \pm 12.2$ & $-4.4 \pm 9.0$ & 0.99 \\
\hline STAIT & $-6.8 \pm 9.8$ & $-3.0 \pm 9.2$ & $-4.2 \pm 12.0$ & 0.27 \\
\hline BDI & $-6.6 \pm 7.4$ & $-4.7 \pm 8.1$ & $-5.0 \pm 7.5$ & 0.51 \\
\hline LDQ & $-5.3 \pm 6.8$ & $-6.1 \pm 7.5$ & $-4.8 \pm 5.9$ & 0.63 \\
\hline PCS & $-10.3 \pm 13.3$ & $-10.1 \pm 13.7$ & $-8.9 \pm 10.1$ & 0.02 \\
\hline ASC & $-1.4 \pm 3.9$ & $-0.9 \pm 5.0$ & $-1.3 \pm 4.6$ & 0.94 \\
\hline
\end{tabular}

SD: standard deviation.

and measure the changes in a clinically meaningful time frame. Undoubtedly, long-term efficacy of treatments should be always the primary outcome in trials. What follows is that short and unpowered studies, including patients whose features may be not representative of those commonly seen in clinical practice, carry the intrinsic risk of drawing biased conclusions. This might have been the case for the efficacy of botulin toxin, which when tested in a solid clinical trial, was not confirmed to provide any additional benefit over acute drug withdrawal (3), as a Cochrane review had anticipated (23).

We chose as the primary outcome of the RCT the proportion of patients with a reduction of at least $50 \%$ in days with migraine at 12-month follow-up, to provide evidence of a concrete and long-lasting effect, and as secondary outcomes the reduction of analgesic intake per month at 6 and 12 months to provide hints of the effect of treatment on MO.

Since acute drug withdrawal should be considered the first step for managing $\mathrm{CM}$ with $\mathrm{MO}$ patients $(2,24)$, we aimed at assessing whether tDCS, which was claimed to have positive effects on the course of the disease $(5,6,25-29)$, had an additional short-, midand long-term effect. To achieve our aim, we compared anodal and cathodal tDCS directly against sham stimulation, and indirectly against acute drug withdrawal alone in patients who did not undergo any pharmacological prophylaxis treatment for the entire study period of 1 year. This latter comparison provided an analysis of efficacy against the standard of care, namely acute drug withdrawal, even though within the longer setting of an RCT that, by definition, differed from the clinical practice. For example, patients enrolled could trust in visits scheduled with intervals shorter than in the common clinical practice and in the availability of access to consultation. This could have partially biased the translational interpretation of RCT results, while offering hints of the opportunity to implement clinical practice with such a personalised pain management approach, even if this required some additional organisational effort.

Pharmacological options for prophylaxis are different, and the drug is generally selected based on patients' clinical history, physical, and psychological comorbidities. On the other hand, pharmacological treatments are sometimes barely tolerated because of their side effects, so the adherence to therapy is often low (30). In the last decades, non-pharmacological methods have been proposed for treating patients with $\mathrm{CM}$, including neuromodulation techniques. Based on the concept that $\mathrm{CM}$ might be associated to central sensitisation, the ability of tDCS to modulate pain-related neural networks has been considered the explanation for the positive findings in small and preliminary studies, for which confirmatory trials have been strongly suggested (14-17,31).

Our study provided robust evidence that tDCS did not change the proportion of patients with $50 \%$ reduction of days of migraine compared to sham stimulation and drug withdrawal alone. Moreover, we found that the monthly reduction both in the number of days of migraine and number of analgesics was significant in all the treatment arms, with no difference between groups. Similarly, all secondary outcome measures that encompassed quality of life, mood and other behavioural and symptom questionnaires improved compared to baseline, with no difference between groups except for catastrophising, which improved better in the tDCS groups compared to the sham group, even though with a score difference that was unlikely to be clinically meaningful. Overall, these results were achieved after acute drug withdrawal by a standardised program of pain management, including behavioural and 
educational measures, short-term scheduled visits, and availability of access to consultation.

Such results at 6 and 12 months appear to be of particular interest considering that the patients, who had failed at least two previous prophylaxis therapies, were not allowed to take any pharmacological prophylaxis for migraine for the entire study period. They have been educated to use abortive medications just in the case of very intense pain attacks, following the standard of care of our Headache Centre. All patients were also educated to apply some behavioural measures to avoid pain or to manage it, such as practising physical activity, having regular meals and regular sleep habits during the period of the study program and follow-up (32). Even though these optional approaches were not standardised and controlled, the compliance of patients to the entire study program, including these behavioural suggestions, could have contributed to the positive outcome.

The improvement of the psychological variables known to influence the course of CM was in keeping with the overall improvement of the somatic symptoms. In particular, all secondary measures of impact of migraine in daily life and activities, anxiety, depression, and catastrophising improved compared to baseline.

The program to which patients could adhere both in the RCT and open-label study was different from the regular clinical practice. Patients were seen regularly every 3 months, and they were free to contact the neurologists any time they needed to be reinforced. Supplemental clinical contacts by phone call or emails were needed in $10 \%$ of cases, whereas three patients needed one supplemental visit. This particular setting of treatment could be important to reinforce the clinical benefit, by increasing patients' confidence in their abilities to manage pain, while ensuring they would be supported by clinicians when needed. A significant percentage of patients with $\mathrm{CM}$ have an overuse of analgesics (33), and it was reported that acute drug withdrawal could also induce the reduction of migraine frequency in the long term $(34,35)$.

In conclusion, our study provided conclusive evidence that tDCS did not influence the course of CM with MO after acute drug withdrawal in the short and long term. It confirmed that acute drug withdrawal is a well-tolerated and effective first-line treatment. Finally, it strengthened the concept that programs including behavioural and educational measures for pain management, short-term scheduled visits and availability of access to consultation could favour long-term improvement and low rate of relapses.

Our study might have some limitations. The openlabel group was not added as one further arm of the double-blind part of the trial. However, results might have been biased by the lack of any active or sham tDCS procedure, and therefore would not have been comparable. Even though the open label group had the intrinsic limitation of a non-parallel analysis, we thought it could have provided a clinically meaningful comparison between the results from the RCT and the current clinical practice, while ensuring the integrity of the RCT. Moreover, patients enrolled could trust in visits scheduled with intervals shorter than in common clinical practice and in the availability of access to consultation. This could have partially biased the translational interpretation of RCT results, while offering hints on the opportunity to implement clinical practice with such a personalised pain management approach, even if it requires some additional organisational effort.

\section{Clinical implications}

- CM patients are difficult to treat, and the regular use of medications can lead to an increased frequency and intensity of migraine attacks. Such association with medication overuse (MO) makes the overall treatment even more challenging.

- The efficacy of neurostimulation techniques, in particular tDCS, in treating migraine has been tested using different approaches in several clinical studies with inconclusive results.

- We designed a placebo-controlled, double-blind, randomised clinical trial (RCT) combined with an ensuing open label study to assess, over 1-year follow-up, short-, mid- and long-term efficacy of tDCS in patients with $\mathrm{CM}$ and $\mathrm{MO}$ after acute drug withdrawal and no migraine prophylaxis.

- Our study provided conclusive evidence that tDCS did not influence the course of CM with MO after acute drug withdrawal in the short and long term, and confirmed that acute drug withdrawal is a well-tolerated and effective first line treatment.

- The study strengthened the concept that tailored programs of pain management, including behavioural and educational measures, short-term scheduled visits and availability of access to consultation could favour long-term improvement and low rate of relapses. 


\section{Declaration of conflicting interests}

The authors declared no potential conflicts of interest with respect to the research, authorship, and/or publication of this article.

\section{Funding}

The authors received no financial support for the research, authorship, and/or publication of this article.

\section{ORCID iD}

Giuseppe Lauria (D) https://orcid.org/0000-0001-9773-020X

\section{References}

1. Headache Classification Committee of the International Headache Society. The International Classification of Headache Disorders, 3rd edition (beta version). Cephalalgia 2013; 33: 629-808.

2. Diener HC, Dodick D, Evers S, et al. Pathophysiology, prevention, and treatment of medication overuse headache. Lancet Neurol 2019; 18: 891-902.

3. Pijpers JA, Kies DA, Louter MA, et al. Acute withdrawal and botulinum toxin $\mathrm{A}$ in chronic migraine with medication overuse: A double-blind randomized controlled trial. Brain 2019; 142: 1203-1214.

4. Grazzi L, Andrasik F, D'Amico D, et al. Disability in chronic migraine patients with medication overuse: Treatment effects at 1-year follow-up. Headache 2004; 44: 678-683.

5. Andrade SM, de Brito Aranha REL, de Oliveira EA, et al. Transcranial direct current stimulation over the primary motor vs prefrontal cortex in refractory chronic migraine: A pilot randomized controlled trial. J Neurol Sci 2017; 378: 225-232.

6. Przeklasa-Muszynska A, Kocot-Kepska M, Dobrogowski J, et al. Transcranial direct current stimulation (tDCS) and its influence on analgesics effectiveness in patients suffering from migraine headache. Pharmacol Rep 2017; 69: 714-721.

7. Lefaucheur JP, Antal A, Ayache SS, et al. Evidencebased guidelines on the therapeutic use of transcranial direct current stimulation (tDCS). Clin Neurophysiol 2017; 128: 56-92.

8. Zortea M, Ramalho L, Alves RL, et al. Transcranial direct current stimulation to improve the dysfunction of descending pain modulatory system related to opioids in chronic non-cancer pain: An integrative review of neurobiology and meta-analysis. Front Neurosci 2019; 13: 1218.

9. Bayer KE, Neeb L, Bayer A, et al. Reduction of intraabdominal pain through transcranial direct current stimulation: A systematic review. Medicine (Baltimore) 2019; 98: e17017.

10. Deus-Yela J, Soler MD, Pelayo-Vergara R, et al. Transcranial direct current stimulation for the treatment of fibromyalgia: A systematic review. Rev Neurol 2017; 65: 353-360.

11. David M, Moraes AA, Costa MLD, et al. Transcranial direct current stimulation in the modulation of neuropathic pain: A systematic review. Neurol Res 2018; 40: 555-563.

12. O'Connell NE, Marston L, Spencer S, et al. Non-invasive brain stimulation techniques for chronic pain. Cochrane Database Syst Rev 2018; 4: CD008208.

13. Rapinesi C, Del Casale A, Scatena P, et al. Add-on deep transcranial magnetic stimulation (dTMS) for the treatment of chronic migraine: A preliminary study. Neurosci Lett 2016; 623: 7-12.

14. Shirahige L, Melo L, Nogueira F, et al. Efficacy of noninvasive brain stimulation on pain control in migraine patients: A systematic review and meta-analysis. Headache 2016; 56: 1565-1596.

15. Stilling JM, Monchi O, Amoozegar F, et al. Transcranial magnetic and direct current stimulation (TMS/tDCS) for the treatment of headache: A systematic review. Headache 2019; 59: 339-357.

16. Puledda F and Goadsby PJ. An update on nonpharmacological neuromodulation for the acute and preventive treatment of migraine. Headache 2017; 57: 685-691.

17. Feng Y, Zhang B, Zhang J, et al. Effects of non-invasive brain stimulation on headache intensity and frequency of headache attacks in patients with migraine: A systematic review and meta-analysis. Headache 2019; 59: 1436-1447.

18. Nielsen M, Carlsen LN, Munksgaard SB, et al. Complete withdrawal is the most effective approach to reduce disability in patients with medication-overuse headache: A randomized controlled open-label trial. Cephalalgia 2019; 39: 863-872.

19. Knotkova H1, Nitsche MA and Cruciani RA. Putative physiological mechanisms underlying tDCS analgesic effects. Front Hum Neurosci 2013; 7: 628.

20. Dasilva AF, Mendonca M, Zaghi S, et al. tDCS-induced analgesia and electrical fields in pain-related neural networks in chronic migraine. Headache 2012; 52: 1283-1295.

21. Feng Y, Zhang B, Zhang J, et al. Effects of non-invasive brain stimulation on headache intensity and frequency of headache attacks in patients with migraine: A systematic review and meta-analysis. Headache 2019; 59: 1436-1447.

22. Luedtke K, Rushton A, Wright C, et al. Effectiveness of transcranial direct current stimulation preceding cognitive behavioural management for chronic low back pain: Sham controlled double blinded randomised controlled trial. BMJ 2015; 350: h1640.

23. Herd CP, Tomlinson CL, Rick C, et al. Botulinum toxins for the prevention of migraine in adults. Cochrane Database Syst Rev 2018; 6: CD011616.

24. Chiang CC, Schwedt TJ, Wang SJ, et al. Treatment of medication-overuse headache: A systematic review. Cephalalgia 2016; 36: 371-386.

25. Cortese F, Pierelli F, Bove I, et al. Anodal transcranial direct current stimulation over the left temporal pole restores normal visual evoked potential habituation in interictal migraineurs. $J$ Headache Pain 2017; 18: 70.

26. Vecchio E, Ricci K, Montemurno A, et al. Effects of left primary motor and dorsolateral prefrontal cortex 
transcranial direct current stimulation on laser-evoked potentials in migraine patients and normal subjects. Neurosci Lett 2016; 626: 149-157.

27. Rocha S, Melo L, Boudoux C, et al. Transcranial direct current stimulation in the prophylactic treatment of migraine based on interictal visual cortex excitability abnormalities: A pilot randomized controlled trial. J Neurol Sci 2015; 349: 33-39.

28. Vigano A, D'Elia TS, Sava SL, et al. Transcranial direct current stimulation (tDCS) of the visual cortex: A proofof-concept study based on interictal electrophysiological abnormalities in migraine. J Headache Pain 2013; 14: 23.

29. Pinchuk D, Pinchuk O, Sirbiladze K, et al. Clinical effectiveness of primary and secondary headache treatment by transcranial direct current stimulation. Front Neurol 2013; 4: 25.

30. Hepp Z, Bloudek LM and Varon SF. Systematic review of migraine prophylaxis adherence and persistence. $J$ Manag Care Pharm 2014; 20: 22-33.
31. Martelletti P, Jensen RH, Antal A, et al. Neuromodulation of chronic headaches: Position statement from the European Headache Federation. J Headache Pain 2013; 14: 86.

32. Grazzi L, Prunesti A and Bussone G. Proposal of a model for multidisciplinary treatment program of chronic migraine with medication overuse: Preliminary study. Neurol Sci 2015; 36: 169-171.

33. Schwedt TJ. Chronic migraine. BMJ 2014; 348: g1416.

34. Pijpers JA, Louter MA, de Bruin ME, et al. Detoxification in medication-overuse headache, a retrospective controlled follow-up study: Does care by a headache nurse lead to cure? Cephalalgia 2016; 36: 122-130.

35. Carlsen LN, Munksgaard SB, Jensen RH, et al. Complete detoxification is the most effective treatment of medication-overuse headache: A randomized controlled open-label trial. Cephalalgia 2018; 38: 225-236. 